\title{
Balancing B-cell subsets in EAE
}

Rituximab, a CD20-specific monoclonal antibody that depletes B cells, has shown promising results in patients with relapsingremitting multiple sclerosis. Studies of experimental autoimmune encephalomyelitis (EAE), a mouse model of multiple sclerosis, have shown that B cells markedly influence the course of disease, but whether their effects are protective or pathological has been a matter of debate. Now, Tedder and colleagues help to resolve this issue by showing that B-cell depletion with a CD20specific antibody has opposing effects on the severity of EAE depending on whether treatment is carried out prior to or following disease initiation.

The authors first investigated how the timing of B-cell depletion influenced the progression of EAE. B-cell depletion 7 days prior to the induction of EAE
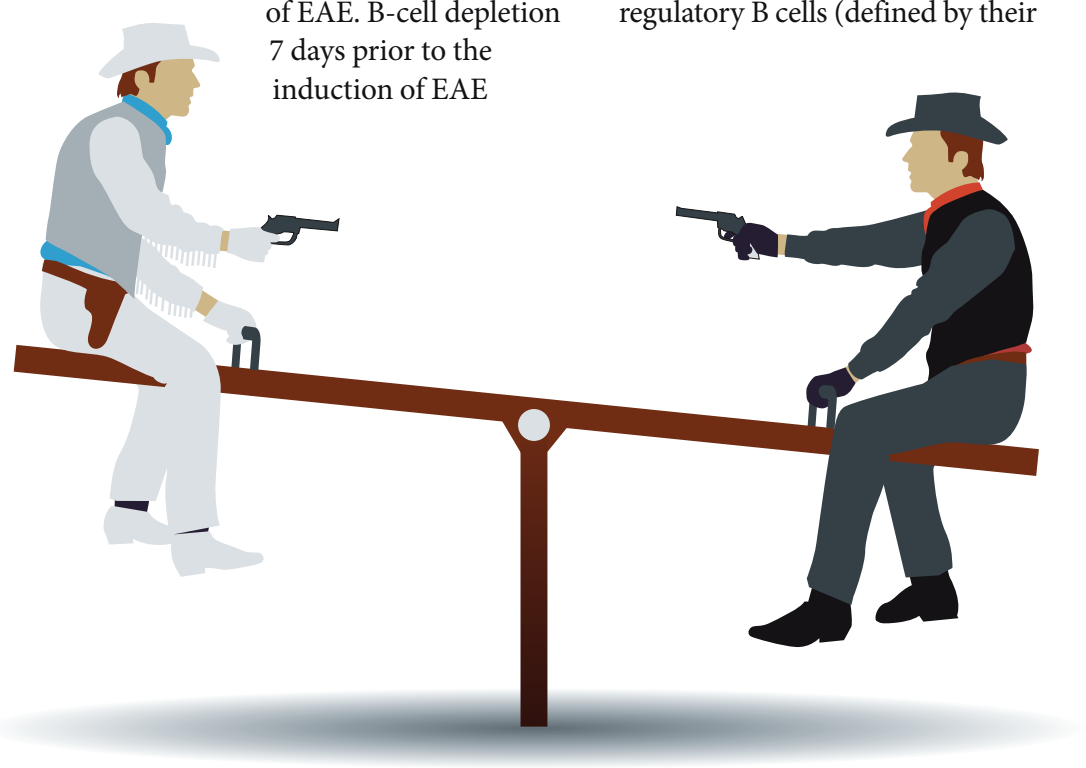

was found to markedly exacerbate disease and was accompanied by a greater degree of immune-cell infiltration of the central nervous system (CNS), by the proliferation of myelin-specific $\mathrm{CD} 4^{+} \mathrm{T}$ cells and by an increase in the number of $\mathrm{CD}^{+}$ $\mathrm{T}$ cells that produced interferon- $\gamma$ and interleukin-17 (IL-17). By contrast, B-cell depletion 14 days after disease induction had the opposite effect: the severity of EAE was significantly reduced compared with control mice, and CD4 $4^{+} \mathrm{T}$-cell proliferation and cytokine production were decreased.

So, what aspects of B-cell function are involved in the observed reciprocal effects on EAE pathology? The severity of EAE did not necessarily correlate with the level of autoantibody production; rather, a rare population of IL-10-producing regulatory $\mathrm{B}$ cells (defined by their
CD $1 \mathrm{~d}^{\text {hi }} \mathrm{CD} 5^{+}$phenotype and referred to as B10 cells) was found to be important for suppressing the initiation of the disease. Adoptive transfer of $\mathrm{CD} 1 \mathrm{~d}^{\text {hi }} \mathrm{CD}^{+} \mathrm{B}$ cells, but not of other B cells, attenuated EAE when transferred before the disease was induced in B-cell depleted mice. However, adoptive transfer of CD1d ${ }^{\text {hi }} \mathrm{CD} 5^{+} \mathrm{B}$ cells had no effect when transferred after the induction of the disease. Instead, the attenuation of disease following B-cell depletion of mice with established EAE was due to the elimination of B cells that supported the expansion of autoreactive $\mathrm{CD} 4^{+}$ T-cell populations in the CNS.

Therefore, at least two different subsets of B cells influence the course of EAE pathology. CD1d ${ }^{\text {hi }} \mathrm{CD} 5^{+}$ $B$ cells limit the onset of disease through IL-10 production, whereas pathogenic B cells contribute to the generation of robust myelin-specific $\mathrm{CD}^{+} \mathrm{T}$-cell responses in established disease. Although it remains to be determined whether $\mathrm{B}$ cells have similar roles in patients with multiple sclerosis, these findings help to expand our understanding of how different B-cell subsets can influence CNS inflammation.

Sarah Allan

ORIGINAL RESEARCH PAPER Matsushita, T

Yanaba, K., Bouaziz, J.-D., Fujimoto, M. \& Tedder, T. F. Regulatory $B$ cells inhibit EAE initiation in mice while other $B$ cells promote disease progression. J. Clin. Invest. 118, 3420-3430 (2008) FURTHER READING Kurosaki, T. Paradox of B cell-targeted therapies. J. Clin. Invest. 118, 3260-3263 (2008) | Fillatreau, S., Gray, D. \& Anderton, S. M. Not always the bad guys: B cells as regulators of autoimmune pathology. Nature Rev. Immunol. 8, 391-397 (2008) 